\title{
GREEN'S FUNCTION, WAVEFUNCTION AND WIGNER FUNCTION OF THE MIC-KEPLER PROBLEM
}

\author{
TOMOYO KANAZAWA
}

Communicated by Ivaïlo M. Mladenov

\begin{abstract}
The phase-space formulation of the nonrelativistic quantum mechanics is constructed on the basis of a deformation of the classical mechanics by the $*$ product. We have taken up the MIC-Kepler problem in which Iwai and Uwano have interpreted its wave-function as the cross section of complex line bundle associated with a principal fibre bundle in the conventional operator formalism. We show that its Green's function, which is derived from the $*$-exponential corresponds to unitary operator through the Weyl application, is equal to the infinite series that consists of its wave-functions. Finally, we obtain its Wigner function.
\end{abstract}

\section{Introduction}

We come to the reluctant conclusion that in our previous paper [5] we obtained only a piece of the local expression of the Green's function for the MIC-Kepler problem. There (Theorem 12) we have presented two expressions denoted by $G_{+}\left(\boldsymbol{r}_{f}, \boldsymbol{r}_{i} ; E\right)$ and $G_{-}\left(\tilde{\boldsymbol{r}}_{f}, \tilde{\boldsymbol{r}}_{i} ; E\right)$ where $\boldsymbol{r}=\tilde{\boldsymbol{r}}$ means the position vector $\boldsymbol{x}$ in $\dot{\mathbb{R}}^{3}=\mathbb{R}^{3} \backslash\{0\}$ i.e., $\boldsymbol{r}=(x, y, z)$. However, $G_{-}\left(\tilde{\boldsymbol{r}}_{f}, \tilde{\boldsymbol{r}}_{i} ; E\right)$ is actually identical with $G_{+}\left(\boldsymbol{r}_{f}, \boldsymbol{r}_{i} ; E\right)$ because the transition function is constant (independent of $\boldsymbol{x}$ ) and therefore, despite the difference in appearance, $\tau_{-}$is essentially the same local trivialization as $\tau_{+}$. This is the reason why $G_{-}\left(\tilde{\boldsymbol{r}}_{f}, \tilde{\boldsymbol{r}}_{i} ; E\right)$ became equivalent to $G_{+}\left(\boldsymbol{r}_{f}, \boldsymbol{r}_{i} ; E\right)$ in the case of iii). After that we have succeeded in obtaining the other piece of the local expression denoted by $G_{-}\left(\boldsymbol{x}_{f}, \boldsymbol{x}_{i} ; E\right)$ via of finding another local trivialization $\tau_{-}$which is transformed into $\tau_{+}$by the transition function of principal $S^{1}$ bundle varying with the position (more precisely, the longitudinal angle) of point $\boldsymbol{x}$ (see [4]). We have found, in addition, the wave-function of the MIC-Kepler problem. In this paper, by turning the right-hand system of orthogonal curvilinear local coordinates on $U_{-}$into the left-hand one, we obtain the Green's function and wave-function in a new form. In this way we end up with two left-handed coordinate systems bringing the two local trivializations which are transformed into each other by the transition function of the principal $S^{1}$ bundle. Thus it becomes possible to obtain its Wigner function on $T^{*}\left(U_{+} \cap U_{-}\right) \subset T^{*} \dot{\mathbb{R}}^{3}$. 
The energy-eigenfunction on the phase space is called Wigner function, and we found that of the MIC-Kepler problem on the reduced phase-space of $T^{*} \dot{\mathbb{R}}^{4}$ by solving the $*$-characteristic equations for its energy and angular momentum where $*$ denotes the Moyal product generated from the canonical coordinates bringing the standard symplectic form on $T^{*} \dot{\mathbb{R}}^{4}$ (see [5, Theorem 10]). How they could be expressed in each of the local coordinate systems on $T^{*} \dot{\mathbb{R}}^{3}$ is an interesting question, which we have succeeded to answer.

The contents of this paper is as follows. In Section 2 we indicate our conclusive results of the Green's function and wave-function for the MIC-Kepler problem, where it comes to be apparent that the Green's function is not a function existing globaly on the configuration space $\dot{\mathbb{R}}^{3}$ but a cross section in the complex line bundles over $\dot{\mathbb{R}}^{3}$ which have been introduced by Iwai and Uwano [3]. In Section 3, we express the Hamiltonian system of the MIC-Kepler problem in terms of the spherical coordinates and their conjugate momentums to obtain its Wigner function on the phase space $T^{*} \dot{\mathbb{R}}^{3}$ without $z$-axis.

\section{Green's Function and Wavefunction}

The MIC-Kepler problem is the reduced Hamiltonian system of the four-dimensional conformal Kepler problem by an $S^{1}$ action, if the associated momentum mapping equals to some fixed value $\mu$ which stands for the strength of Dirac's monopole field [2]. Then the Green's function of the MIC-Kepler problem is obtained by reducing that one of the conformal Kepler problem which have been already shown in [4] and [5]. Here we have found another kind of local coordinate system especially reconsidering one side of the local trivializations, denoted by $\tau_{-}$, derived from the open subset $U_{-}$in $\dot{\mathbb{R}}^{3}=U_{+} \cup U_{-}$as it is necessary to make it transformable into the other side $\tau_{+}$through the transition function of the principal fibre bundle $g_{-+}: U_{+} \cap U_{-} \ni \boldsymbol{x} \longmapsto \mathrm{e}^{\mathrm{i} \phi(\boldsymbol{x})}=\mathrm{e}^{-\mathrm{i} \tilde{\phi}(\boldsymbol{x})} \in S^{1}$ (see [4]) and it is also necessary to alter the orientation of the orthogonal curvilinear local coordinate system on $U_{-}$as that on one $U_{+}$i.e., anti-clockwise. The details of the latest local coordinate system are as follows (see Fig. 1).

Let $U_{+}$be an open subset without negative $z$-axis such that

$$
U_{+}=\left\{\boldsymbol{x}(r, \theta, \phi) \in \dot{\mathbb{R}}^{3} ; r>0,0 \leq \theta<\pi, 0 \leq \phi<2 \pi\right\}
$$

bringing the following local trivialization where $\pi$ is the bundle projection and $\nu$ has the range of values $0 \leq \nu<4 \pi$

$\tau_{+}: \pi^{-1}\left(U_{+}\right) \ni \boldsymbol{u} \longmapsto\left(\pi(\boldsymbol{u}), \varphi_{+}(\boldsymbol{u})\right)=(\boldsymbol{x}(r, \theta, \phi), \exp (\mathrm{i} \nu / 2)) \in U_{+} \times S^{1}$ 

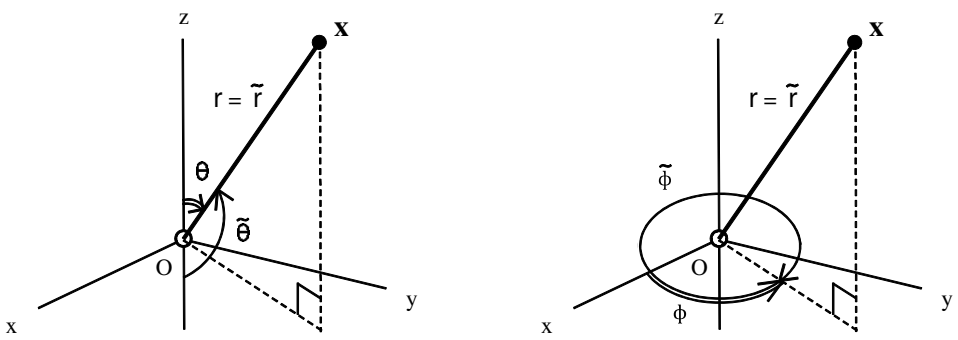

Figure 1. The configuration space $\dot{\mathbb{R}}^{3}=\mathbb{R}^{3} \backslash\{0\}$.

$x=r \sin \theta \cos \phi$
$y=r \sin \theta \sin \phi$
$z=r \cos \theta$$\quad\left\{\begin{aligned} u_{1}=\sqrt{r} \cos \frac{\theta}{2} \cos \frac{\nu+\phi}{2}, & u_{2}=\sqrt{r} \cos \frac{\theta}{2} \sin \frac{\nu+\phi}{2} \\ u_{3}=\sqrt{r} \sin \frac{\theta}{2} \cos \frac{\nu-\phi}{2}, & u_{4}=\sqrt{r} \sin \frac{\theta}{2} \sin \frac{\nu-\phi}{2} .\end{aligned}\right.$

Similarly $U_{-}$is an other open subset without positive $z$-axis such that

$$
U_{-}=\left\{\boldsymbol{x}(\tilde{r}, \tilde{\theta}, \tilde{\phi}) \in \dot{\mathbb{R}}^{3} ; \tilde{r}>0,0 \leq \tilde{\theta}<\pi, 0<\tilde{\phi} \leq 2 \pi\right\}
$$

bringing the following local trivialization where $0 \leq \tilde{\nu}<4 \pi$

$$
\begin{aligned}
& \tau_{-}: \pi^{-1}\left(U_{-}\right) \ni \boldsymbol{u} \longmapsto\left(\pi(\boldsymbol{u}), \varphi_{-}(\boldsymbol{u})\right)=(\boldsymbol{x}(\tilde{r}, \tilde{\theta}, \tilde{\phi}), \exp (\mathrm{i} \tilde{\nu} / 2)) \in U_{-} \times S^{1} \\
& \begin{array}{l}
x=\tilde{r} \sin \tilde{\theta} \cos \tilde{\phi} \\
y=-\tilde{r} \sin \tilde{\theta} \sin \tilde{\phi} \\
z=-\tilde{r} \cos \tilde{\theta}
\end{array}\left\{\begin{array}{l}
u_{1}=-\sqrt{\tilde{r}} \sin \frac{\tilde{\theta}}{2} \cos \frac{\tilde{\nu}+\tilde{\phi}}{2}, \quad u_{2}=-\sqrt{\tilde{r}} \sin \frac{\tilde{\theta}}{2} \sin \frac{\tilde{\nu}+\tilde{\phi}}{2} \\
u_{3}=-\sqrt{\tilde{r}} \cos \frac{\tilde{\theta}}{2} \cos \frac{\tilde{\nu}+3 \tilde{\phi}}{2}, u_{4}=-\sqrt{\tilde{r}} \cos \frac{\tilde{\theta}}{2} \sin \frac{\tilde{\nu}+3 \tilde{\phi}}{2} .
\end{array}\right.
\end{aligned}
$$

We suppose that the real parameter $E<0$ denoting the energy of the MIC-Kepler problem do not coinside with some of its eigenvalues as given in [6] and [5, Theorem 10]

$$
E \neq E_{N}=-\frac{2 m k^{2}}{\hbar^{2}(N+2)^{2}}, \quad N=0,1,2, \ldots
$$

The positive constant $m$ is the mass of the electron. The charge of the electron equals $-e$ where $e>0$ is the charge of the proton called an 'elementary charge', and $k$ is the positive constant defined as

$$
k \equiv \frac{e^{2}}{4 \pi \varepsilon}
$$


where $\varepsilon>0$ means electric permittivity. The positive parameter $\hbar$ is defined as $\hbar \equiv h / 2 \pi$ where $h$ is Planck's constant. Then we reduce the Green's function of the conformal Kepler problem denoted by $G\left(\boldsymbol{u}_{f}, \boldsymbol{u}_{i} ; 4 k\right)$ to the Green's functions of the MIC-Kepler problem on $U_{+}$and $U_{-}$denoted by $G_{+}\left(\boldsymbol{x}_{f}, \boldsymbol{x}_{i} ; E\right)$ and $G_{-}\left(\boldsymbol{x}_{f}, \boldsymbol{x}_{i} ; E\right)$ respectively. The subscripts $i$ and $f$ say that the points with each of them denote initial and final points of motion in a proper configuration space respectively. The final results are in the following proposition where $l$ is an arbitrary integer quantizing $\mu$ by the relation of $\mu=l \hbar / 2$, and $J_{l}(v)$ denote the Bessel functions.

Proposition 1. i) When $\boldsymbol{x}_{i}, \boldsymbol{x}_{f} \in U_{+}$, the Green's function of the MIC-Kepler problem is

$$
\begin{aligned}
G_{+}\left(\boldsymbol{x}_{f}, \boldsymbol{x}_{i} ; E=\right. & \left.-m \omega^{2} / 8\right) \\
= & r_{f} \lim _{\chi \rightarrow 4 \pi-0} \int_{0}^{\chi} G\left(\boldsymbol{u}_{f}, \boldsymbol{u}_{i} ; 4 k\right) \exp \left(\mathrm{i} l \frac{\nu_{i}-\nu_{f}}{2}\right) \mathrm{d} \nu_{i} \\
= & -\frac{\mathrm{i}^{l+1} m^{2} \omega^{2}}{16 \pi \hbar^{3}} \lim _{y^{\prime} \rightarrow+0} \int_{0}^{\infty} \mathrm{e}^{-\frac{\mathrm{i}}{\hbar}\left(4 k-\mathrm{i} y^{\prime}\right)\left(t+\mathrm{i} y^{\prime}\right)} \operatorname{cosec}^{2}\left(\omega t+\mathrm{i} \omega y^{\prime}\right) \\
& \times \exp \left[-\mathrm{i} \frac{m \omega}{2 \hbar}\left(r_{i}+r_{f}\right) \cot \left(\omega t+\mathrm{i} \omega y^{\prime}\right)-\mathrm{i} l \cdot \frac{\Theta}{2}\right] \\
& \times J_{l}\left(\frac{m \omega}{2 \hbar} \sqrt{2 \boldsymbol{x}_{i} \cdot \boldsymbol{x}_{f}+2 r_{i} r_{f}} \operatorname{cosec}\left(\omega t+\mathrm{i} \omega y^{\prime}\right)\right) \mathrm{d} t \\
\text { where } \frac{\Theta}{2} \equiv \tan ^{-1} & {\left[\frac{\sin \frac{\phi_{i}-\phi_{f}}{2}}{\cos \frac{\phi_{i}-\phi_{f}}{2}} \cdot \frac{\cos \frac{\theta_{i}+\theta_{f}}{2}}{\cos \frac{\theta_{i}-\theta_{f}}{2}}\right] . }
\end{aligned}
$$

ii) When $\boldsymbol{x}_{i}, \boldsymbol{x}_{f} \in U_{-}$, the Green's function is

$$
\begin{aligned}
G_{-}\left(\boldsymbol{x}_{f}, \boldsymbol{x}_{i} ; E=\right. & \left.-m \omega^{2} / 8\right) \\
= & \tilde{r}_{f} \lim _{\chi \rightarrow 4 \pi-0} \int_{0}^{\chi} G\left(\boldsymbol{u}_{f}, \boldsymbol{u}_{i} ; 4 k\right) \exp \left(\mathrm{i} l \frac{\tilde{\nu}_{i}-\tilde{\nu}_{f}}{2}\right) \mathrm{d} \tilde{\nu}_{i} \\
= & -\frac{\mathrm{i}^{l+1} m^{2} \omega^{2}}{16 \pi \hbar^{3}} \lim _{y^{\prime} \rightarrow+0} \int_{0}^{\infty} \mathrm{e}^{-\frac{\mathrm{i}}{\hbar}\left(4 k-\mathrm{i} y^{\prime}\right)\left(t+\mathrm{i} y^{\prime}\right)} \operatorname{cosec}^{2}\left(\omega t+\mathrm{i} \omega y^{\prime}\right) \\
& \times \exp \left[-\mathrm{i} \frac{m \omega}{2 \hbar}\left(\tilde{r}_{i}+\tilde{r}_{f}\right) \cot \left(\omega t+\mathrm{i} \omega y^{\prime}\right)-\mathrm{i} l \cdot \frac{\tilde{\Theta}}{2}\right] \\
& \times J_{l}\left(\frac{m \omega}{2 \hbar} \sqrt{2 \boldsymbol{x}_{i} \cdot \boldsymbol{x}_{f}+2 \tilde{r}_{i} \tilde{r}_{f}} \operatorname{cosec}\left(\omega t+\mathrm{i} \omega y^{\prime}\right)\right) \mathrm{d} t
\end{aligned}
$$


where

$$
\frac{\tilde{\Theta}}{2} \equiv \tan ^{-1}\left[\frac{\sin \frac{\tilde{\phi}_{i}-\tilde{\phi}_{f}}{2}}{\cos \frac{\tilde{\phi}_{i}-\tilde{\phi}_{f}}{2}} \cdot \frac{2 \cos \left(\tilde{\phi}_{i}-\tilde{\phi}_{f}\right) \cos \frac{\tilde{\theta}_{i}}{2} \cos \frac{\tilde{\theta}_{f}}{2}+\cos \frac{\tilde{\theta}_{i}-\tilde{\theta}_{f}}{2}}{2 \cos \left(\tilde{\phi}_{i}-\tilde{\phi}_{f}\right) \cos \frac{\tilde{\theta}_{i}}{2} \cos \frac{\tilde{\theta}_{f}}{2}-\cos \frac{\tilde{\theta}_{i}+\tilde{\theta}_{f}}{2}}\right] .
$$

iii) When $\boldsymbol{x}_{i}, \boldsymbol{x}_{f} \in U_{+} \cap U_{-}, \theta=\pi-\tilde{\theta}, \phi=2 \pi-\tilde{\phi}$ and $\tilde{\Theta}$ is given by

$$
\frac{\tilde{\Theta}}{2}=\tan ^{-1}\left[-\frac{\sin \frac{\phi_{i}-\phi_{f}}{2}}{\cos \frac{\phi_{i}-\phi_{f}}{2}} \cdot \frac{2 \cos \left(\phi_{i}-\phi_{f}\right) \sin \frac{\theta_{i}}{2} \sin \frac{\theta_{f}}{2}+\cos \frac{\theta_{i}-\theta_{f}}{2}}{2 \cos \left(\phi_{i}-\phi_{f}\right) \sin \frac{\theta_{i}}{2} \sin \frac{\theta_{f}}{2}+\cos \frac{\theta_{i}+\theta_{f}}{2}}\right] .
$$

As it can be seen, the Green's functions on $U_{+} \cap U_{-}$are not equivalent with each other since the difference between $G_{+}$and $G_{-}$is the one between $\Theta$ and $\tilde{\Theta}$ because of the equality $r=\tilde{r}$. Besides $\tilde{\Theta}$ is not equal to $\Theta$ obviously. Right then, what is the concrete relation between $G_{+}$and $G_{-}$on the common part? An answer to this question is the following proposition established by similar methods as that in the earlier paper $[4, \S 5]$.

Proposition 2. i) When $\boldsymbol{x} \in U_{+}$, the wave function of the MIC-Kepler problem is

$$
\begin{aligned}
\Psi_{N}^{+}(\boldsymbol{x})= & \frac{m \omega}{2 \sqrt{\pi} \hbar}\left(\sqrt{\frac{m \omega}{\hbar}}\right)^{N} \frac{\mathcal{P}\left(r \cos ^{2} \frac{\theta}{2}, r \sin ^{2} \frac{\theta}{2}\right)}{\sqrt{k_{1} ! k_{2} ! k_{3} ! k_{4} !}} \exp \left(-\frac{m \omega}{2 \hbar} r\right) \\
& \left(\sqrt{r} \cos \frac{\theta}{2}\right)^{k_{1}+k_{3}}\left(\sqrt{r} \sin \frac{\theta}{2}\right)^{k_{2}+k_{4}} \exp \left[-\mathrm{i}\left(k_{1}-k_{2}-k_{3}+k_{4}\right) \frac{\phi}{2}\right]
\end{aligned}
$$

and if $\boldsymbol{x}_{i}, \boldsymbol{x}_{f} \in U_{+}$, the Green's function of the MIC-Kepler problem is also written by

$$
G_{+}\left(\boldsymbol{x}_{f}, \boldsymbol{x}_{i} ; E\right)=\sum_{N=0}^{\infty} \frac{1}{4 k-(N+2) \hbar \omega} \Psi_{N}^{+}\left(\boldsymbol{x}_{f}\right) \overline{\Psi_{N}^{+}\left(\boldsymbol{x}_{i}\right)}
$$

where $\omega \equiv \sqrt{-8 E / m}$, the infinite sum of $N$ includes the finite sum of all terms whose non-negative integers $\left(k_{1}, k_{2}, k_{3}, k_{4}\right)$ that satisfy the following conditions for a fixed $N \in \mathbb{N} \cup\{0\}$

$$
k_{1}+k_{2}+k_{3}+k_{4}=N, \quad k_{1}+k_{2}-k_{3}-k_{4}=-l, \quad \mathbb{Z} \ni l=2 \mu / \hbar
$$

and $\mathcal{P}(X, Y)$ is the polynomial

$$
\mathcal{P}(X, Y)=\sum_{j=0}^{k_{1}} \sum_{s=0}^{k_{2}} j ! s !\left(-\frac{\hbar}{m \omega}\right)^{j+s}{ }_{k_{1}} C_{j} \cdot{ }_{k_{3}} C_{j} \cdot k_{2} C_{s} \cdot{ }_{k_{4}} C_{s} X^{-j} Y^{-s} .
$$


ii) When $\boldsymbol{x} \in U_{-}$, the wave function of the MIC-Kepler problem is

$$
\begin{aligned}
\Psi_{N}^{-}(\boldsymbol{x})= & \frac{m \omega}{2 \sqrt{\pi} \hbar}\left(-\sqrt{\frac{m \omega}{\hbar}}\right)^{N} \frac{\mathcal{P}\left(\tilde{r} \sin ^{2} \frac{\tilde{\theta}}{2}, \tilde{r} \cos ^{2} \frac{\tilde{\theta}}{2}\right)}{\sqrt{k_{1} ! k_{2} ! k_{3} ! k_{4} !}} \exp \left(-\frac{m \omega}{2 \hbar} \tilde{r}\right) \\
& \left(\sqrt{\tilde{r}} \sin \frac{\tilde{\theta}}{2}\right)^{k_{1}+k_{3}}\left(\sqrt{\tilde{r}} \cos \frac{\tilde{\theta}}{2}\right)^{k_{2}+k_{4}} \exp \left[-\mathrm{i}\left(k_{1}+3 k_{2}-k_{3}-3 k_{4}\right) \frac{\tilde{\phi}}{2}\right]
\end{aligned}
$$

and for $\boldsymbol{x}_{i}, \boldsymbol{x}_{f} \in U_{-}$, the Green's function of the MIC-Kepler problem is

$$
G_{-}\left(\boldsymbol{x}_{f}, \boldsymbol{x}_{i} ; E\right)=\sum_{N=0}^{\infty} \frac{1}{4 k-(N+2) \hbar \omega} \Psi_{N}^{-}\left(\boldsymbol{x}_{f}\right) \bar{\Psi}_{N}^{-}\left(\boldsymbol{x}_{i}\right)
$$

iii) When $\boldsymbol{x} \in U_{+} \cap U_{-}$, the relation between $\Psi_{N}^{+}$and $\Psi_{N}^{-}$is

$$
\Psi_{N}^{-}(\boldsymbol{x})=\Psi_{N}^{+}(\boldsymbol{x}) \mathrm{e}^{-\mathrm{i} l \phi}
$$

and if $\boldsymbol{x}_{i}, \boldsymbol{x}_{f} \in U_{+} \cap U_{-}$, the relation between $G_{+}$and $G_{-}$is

$$
G_{-}\left(\boldsymbol{x}_{f}, \boldsymbol{x}_{i} ; E\right)=G_{+}\left(\boldsymbol{x}_{f}, \boldsymbol{x}_{i} ; E\right) \mathrm{e}^{-\mathrm{i} l\left(\phi_{f}-\phi_{i}\right)} .
$$

\section{Wigner Function}

When the $z$-axis is excluded from the configuration space $\dot{\mathbb{R}}^{3}$ the Hamiltonian system of the conformal Kepler problem $\left(T^{*}\left(\pi^{-1}\left(U_{+} \cap U_{-}\right)\right)\right.$, $\left.\mathrm{d} \vartheta, H\right)$ where $\vartheta$ denotes the canonical one-form of $T^{*} \dot{\mathbb{R}}^{4} \supset T^{*}\left(\pi^{-1}\left(U_{+} \cap U_{-}\right)\right)$is described in the above-mentioned local trivializations $\tau_{+}$and $\tau_{-}$(see also $§ 2$ ) as follows ${ }^{1}$

$H\left(r, \theta, \phi, \nu, \rho_{r}, \rho_{\theta}, \rho_{\phi}, \rho_{\nu}\right)=\frac{1}{2 m}\left(\rho_{r}^{2}+\frac{\rho_{\theta}^{2}}{r^{2}}+\frac{\rho_{\phi}^{2}+\rho_{\nu}^{2}-2 \rho_{\phi} \rho_{\nu} \cos \theta}{r^{2} \sin ^{2} \theta}\right)-\frac{k}{r}$

where $\rho_{r}, \rho_{\theta}, \rho_{\phi}$ and $\rho_{\nu}$ are the conjugate momentums of $r, \theta, \phi$ and $\nu$ respectively such that $\vartheta \equiv \rho_{1} \mathrm{~d} u_{1}+\rho_{2} \mathrm{~d} u_{2}+\rho_{3} \mathrm{~d} u_{3}+\rho_{4} \mathrm{~d} u_{4}=\rho_{r} \mathrm{~d} r+\rho_{\theta} \mathrm{d} \theta+\rho_{\phi} \mathrm{d} \phi+\rho_{\nu} \mathrm{d} \nu$, or

$$
\begin{aligned}
& H\left(\tilde{r}, \tilde{\theta}, \tilde{\phi}, \tilde{\nu}, \rho_{\tilde{r}}, \rho_{\tilde{\theta}}, \rho_{\tilde{\phi}}, \rho_{\tilde{\nu}}\right) \\
& \quad=\frac{1}{2 m}\left(\rho_{\tilde{r}}^{2}+\frac{\rho_{\tilde{\theta}}^{2}}{\tilde{r}^{2}}+\frac{\rho_{\tilde{\phi}}^{2}+\rho_{\tilde{\nu}}^{2}(4 \cos \tilde{\theta}+5)-2 \rho_{\tilde{\phi}} \rho_{\tilde{\nu}}(\cos \tilde{\theta}+2)}{\tilde{r}^{2} \sin ^{2} \tilde{\theta}}\right)-\frac{k}{\tilde{r}}
\end{aligned}
$$

\footnotetext{
${ }^{1}$ The forms written by the trivialization $\tau_{+}$equal the ones given by Iwai $[1, \S .3]$.
} 
where $\rho_{\tilde{r}}, \rho_{\tilde{\theta}}, \rho_{\tilde{\phi}}$ and $\rho_{\tilde{\nu}}$ are the conjugate momentums of $\tilde{r}, \tilde{\theta}, \tilde{\phi}$ and $\tilde{\nu}$ respectively such that $\vartheta \equiv \rho_{1} \mathrm{~d} u_{1}+\rho_{2} \mathrm{~d} u_{2}+\rho_{3} \mathrm{~d} u_{3}+\rho_{4} \mathrm{~d} u_{4}=\rho_{\tilde{r}} \mathrm{~d} \tilde{r}+\rho_{\tilde{\theta}} \mathrm{d} \tilde{\theta}+\rho_{\tilde{\phi}} \mathrm{d} \tilde{\phi}+\rho_{\tilde{\nu}} \mathrm{d} \tilde{\nu}$. Moreover verifying the following equalities

$$
\begin{aligned}
& \tilde{r}=r, \quad \tilde{\theta}+\theta=\pi, \quad \tilde{\phi}+\phi=2 \pi, \quad \tilde{\nu}-\nu=2 \phi \\
& \rho_{\tilde{r}}=\rho_{r}, \quad \rho_{\tilde{\theta}}=-\rho_{\theta}, \quad \rho_{\tilde{\phi}}=2 \rho_{\nu}-\rho_{\phi}, \quad \rho_{\tilde{\nu}}=\rho_{\nu}
\end{aligned}
$$

we have the following equivalence on the common part $T^{*}\left(\pi^{-1}\left(U_{+} \cap U_{-}\right)\right) \subset$ $T^{*} \dot{\mathbb{R}}^{4}$

$$
H\left(r, \theta, \phi, \nu, \rho_{r}, \rho_{\theta}, \rho_{\phi}, \rho_{\nu}\right)=H\left(\tilde{r}, \tilde{\theta}, \tilde{\phi}, \tilde{\nu}, \rho_{\tilde{r}}, \rho_{\tilde{\theta}}, \rho_{\tilde{\phi}}, \rho_{\tilde{\nu}}\right)
$$

Additionally $\rho_{\tilde{\nu}}=\rho_{\nu}$ coincides with the associated momentum mapping $\psi(\boldsymbol{u}, \boldsymbol{\rho})$

$$
\rho_{\tilde{\nu}}=\rho_{\nu}=\frac{1}{2}\left(-u_{2} \rho_{1}+u_{1} \rho_{2}-u_{4} \rho_{3}+u_{3} \rho_{4}\right)=\psi(\boldsymbol{u}, \boldsymbol{\rho})
$$

and therefore restricting the Hamiltonian system $\left(T^{*}\left(\pi^{-1}\left(U_{+} \cap U_{-}\right)\right), \mathrm{d} \vartheta, H\right)$ on the subset $\psi^{-1}(\mu) \subset T_{\boldsymbol{u}}^{*} \dot{\mathbb{R}}^{4}$

$$
\psi^{-1}(\mu)=\left\{(\boldsymbol{u}, \boldsymbol{\rho}) \in T_{\boldsymbol{u}}^{*} \dot{\mathbb{R}}^{4} ; \psi(\boldsymbol{u}, \boldsymbol{\rho})=\mu\right\}
$$

is easily done by setting each of the conjugate momentum $\rho_{\nu}$ or $\rho_{\tilde{\nu}}$ to the fixed value $\mu$. Further, according to Iwai \& Uwano, $T^{*} \dot{\mathbb{R}}^{3}$ is diffeomorphic with the quotient space $\psi^{-1}(\mu) / S^{1}$ (see [2, Lemma 2.4]) i.e., $\pi_{\mu}^{*} \sigma_{\mu}=\iota_{\mu}^{*} \mathrm{~d} \vartheta$ and $\pi_{\mu}^{*} H_{\mu}=$ $\iota_{\mu}^{*} H$ where $\iota_{\mu}: \psi^{-1}(\mu) \rightarrow T^{*} \dot{\mathbb{R}}^{4}$ is the inclusion and $\pi_{\mu}: \psi^{-1}(\mu) \ni(\boldsymbol{u}, \boldsymbol{\rho}) \rightarrow$ $(\boldsymbol{x}, \boldsymbol{p}) \in T^{*} \dot{\mathbb{R}}^{3}$ is the map which provides a principal $S^{1}$ bundle such that $\boldsymbol{x}=$ $\pi(\boldsymbol{u})$ and

$$
\left(\begin{array}{c}
p_{x} \\
p_{y} \\
p_{z} \\
-\mu / r
\end{array}\right)=\frac{1}{4 r}\left(\begin{array}{rrrr}
2 u_{3} & 2 u_{4} & 2 u_{1} & 2 u_{2} \\
-2 u_{4} & 2 u_{3} & 2 u_{2} & -2 u_{1} \\
2 u_{1} & 2 u_{2} & -2 u_{3} & -2 u_{4} \\
2 u_{2} & -2 u_{1} & 2 u_{4} & -2 u_{3}
\end{array}\right)\left(\begin{array}{c}
\rho_{1} \\
\rho_{2} \\
\rho_{3} \\
\rho_{4}
\end{array}\right)
$$

Then, we have the following equations

$$
\begin{array}{lll}
\rho_{r}=p_{r}, & \rho_{\theta}=p_{\theta}, & \rho_{\phi}=p_{\phi}+\mu \cos \theta \\
\rho_{\tilde{r}}=p_{\tilde{r}}, & \rho_{\tilde{\theta}}=p_{\tilde{\theta}}, & \rho_{\tilde{\phi}}=p_{\tilde{\phi}}+\mu \cos \tilde{\theta}+2 \mu
\end{array}
$$

where $p_{r}, p_{\theta}, p_{\phi}, p_{\tilde{r}}, p_{\tilde{\theta}}$ and $p_{\tilde{\phi}}$ are the conjugate momentums of $r, \theta, \phi, \tilde{r}, \tilde{\theta}$ and $\tilde{\phi}$ defined by

$$
p_{x} \mathrm{~d} x+p_{y} \mathrm{~d} y+p_{z} \mathrm{~d} z=p_{r} \mathrm{~d} r+p_{\theta} \mathrm{d} \theta+p_{\phi} \mathrm{d} \phi=p_{\tilde{r}} \mathrm{~d} \tilde{r}+p_{\tilde{\theta}} \mathrm{d} \tilde{\theta}+p_{\tilde{\phi}} \mathrm{d} \tilde{\phi} .
$$


In this way the reduced Hamiltonian system $\left(T^{*}\left(U_{+} \cap U_{-}\right), \sigma_{\mu}, H_{\mu}\right)$ that is referred from now on as the MIC-Kepler problem is

$$
\left\{\begin{array}{l}
\sigma_{\mu}=\mathrm{d} p_{r} \wedge \mathrm{d} r+\mathrm{d} p_{\theta} \wedge \mathrm{d} \theta+\mathrm{d}\left(p_{\phi}+\mu \cos \theta\right) \wedge \mathrm{d} \phi \\
H_{\mu}\left(r, \theta, \phi, p_{r}, p_{\theta}, p_{\phi}\right)=\frac{1}{2 m}\left(p_{r}^{2}+\frac{p_{\theta}^{2}}{r^{2}}+\frac{p_{\phi}^{2}}{r^{2} \sin ^{2} \theta}\right)-\frac{k}{r}+\frac{\mu^{2}}{2 m r^{2}}
\end{array}\right.
$$

or

$$
\left\{\begin{array}{l}
\sigma_{\mu}=\mathrm{d} p_{\tilde{r}} \wedge \mathrm{d} \tilde{r}+\mathrm{d} p_{\tilde{\theta}} \wedge \mathrm{d} \tilde{\theta}+\mathrm{d}\left(p_{\tilde{\phi}}+\mu \cos \tilde{\theta}+2 \mu\right) \wedge \mathrm{d} \tilde{\phi} \\
H_{\mu}\left(\tilde{r}, \tilde{\theta}, \tilde{\phi}, p_{\tilde{r}}, p_{\tilde{\theta}}, p_{\tilde{\phi}}\right)=\frac{1}{2 m}\left(p_{\tilde{r}}^{2}+\frac{p_{\tilde{\theta}}^{2}}{\tilde{r}^{2}}+\frac{p_{\tilde{\phi}}^{2}}{\tilde{r}^{2} \sin ^{2} \tilde{\theta}}\right)-\frac{k}{\tilde{r}}+\frac{\mu^{2}}{2 m \tilde{r}^{2}}
\end{array}\right.
$$

Moreover provided that the following equalities

$$
p_{\tilde{r}}=p_{r}, \quad p_{\tilde{\theta}}=-p_{\theta}, \quad p_{\tilde{\phi}}=-p_{\phi}
$$

are satisfied we have the following equivalence on the common part $T^{*}\left(U_{+} \cap\right.$ $\left.U_{-}\right) \subset T^{*} \dot{\mathbb{R}}^{3}$.

$$
H_{\mu}\left(r, \theta, \phi, p_{r}, p_{\theta}, p_{\phi}\right)=H_{\mu}\left(\tilde{r}, \tilde{\theta}, \tilde{\phi}, p_{\tilde{r}}, p_{\tilde{\theta}}, p_{\tilde{\phi}}\right)
$$

Similarly, the Wigner function of the MIC-Kepler problem (see [5, Theorem 10]) can be rewritten as a function on $T^{*}\left(U_{+} \cap U_{-}\right)$which is almost the phase space $T^{*} \dot{\mathbb{R}}^{3}$. Finally if $N$ is an arbitrary non-negative integer called 'principal quantum number', $l$ is an arbitrary integer quantizing $\mu$ by the relation of $\mu=l \hbar / 2$ and $L_{n}(X)$ denotes the Laguerre polynomial of degree $n$, i.e.,

$$
\begin{aligned}
L_{n}(X) & =\sum_{\alpha=0}^{n}(-1)^{\alpha} \frac{n !}{(\alpha !)^{2}(n-\alpha) !} X^{\alpha} \\
\sum_{n=0}^{\infty} L_{n}(X) \xi^{n} & =\frac{1}{1-\xi} \exp \left(-\frac{\xi}{1-\xi} X\right)
\end{aligned}
$$

we can formulate the following

Proposition 3. Suppose that the point $\boldsymbol{x} \in \mathbb{R}^{3}$ is not on the z-axis. Then the Wigner function of the MIC-Kepler problem is given as follows

$$
\begin{aligned}
& f_{N}\left(r, \theta, \phi, p_{r}, p_{\theta}, p_{\phi}\right)=\frac{(-1)^{N}}{(\pi \hbar)^{4}} \mathrm{e}^{-2(N+2)} \\
& \times L_{n_{a}}\left(\frac{N+2}{2 m k}\left[\mathcal{A}^{2}+\frac{\mathcal{C}^{2}}{r(1+\cos \theta)}\right]\right) L_{n_{b}}\left(\frac{N+2}{2 m k}\left[\mathcal{A}^{2}+\frac{\mathcal{D}^{2}}{r(1+\cos \theta)}\right]\right) \\
& \times L_{n_{c}}\left(\frac{N+2}{2 m k}\left[\mathcal{B}^{2}+\frac{\mathcal{E}^{2}}{r(1-\cos \theta)}\right]\right) L_{n_{d}}\left(\frac{N+2}{2 m k}\left[\mathcal{B}^{2}+\frac{\mathcal{F}^{2}}{r(1-\cos \theta)}\right]\right)
\end{aligned}
$$


or

$$
\begin{aligned}
& f_{N}\left(\tilde{r}, \tilde{\theta}, \tilde{\phi}, p_{\tilde{r}}, p_{\tilde{\theta}}, p_{\tilde{\phi}}\right)=\frac{(-1)^{N}}{(\pi \hbar)^{4}} \mathrm{e}^{-2(N+2)} \\
\times & L_{n_{a}}\left(\frac{N+2}{2 m k}\left[\tilde{\mathcal{A}}^{2}+\frac{\tilde{\mathcal{C}}^{2}}{\tilde{r}(1-\cos \tilde{\theta})}\right]\right) L_{n_{b}}\left(\frac{N+2}{2 m k}\left[\tilde{\mathcal{A}}^{2}+\frac{\tilde{\mathcal{D}}^{2}}{\tilde{r}(1-\cos \tilde{\theta})}\right]\right) \\
\times & L_{n_{c}}\left(\frac{N+2}{2 m k}\left[\tilde{\mathcal{B}}^{2}+\frac{\tilde{\mathcal{E}}^{2}}{\tilde{r}(1+\cos \tilde{\theta})}\right]\right) L_{n_{d}}\left(\frac{N+2}{2 m k}\left[\tilde{\mathcal{B}}^{2}+\frac{\tilde{\mathcal{F}}^{2}}{\tilde{r}(1+\cos \tilde{\theta})}\right]\right)
\end{aligned}
$$

where $n_{a}, n_{b}, n_{c}$ and $n_{d}$ are non-negative integers such that

$$
\left\{\begin{array} { l } 
{ 2 ( n _ { a } + n _ { d } ) = N + l } \\
{ 2 ( n _ { b } + n _ { c } ) = N - l }
\end{array} \text { i.e., } \quad \left\{\begin{array}{l}
|l| \leq N \\
N \text { and l are simultaneously even or odd }
\end{array}\right.\right.
$$

where $\mathcal{A}, \mathcal{B}, \mathcal{C}, \mathcal{D}, \mathcal{E}, \mathcal{F}, \tilde{\mathcal{A}}, \tilde{\mathcal{B}}, \tilde{\mathcal{C}}, \tilde{\mathcal{D}}, \tilde{\mathcal{E}}$ and $\tilde{\mathcal{F}}$ are given by the formulas

$$
\begin{aligned}
& \mathcal{A}\left(r, \theta, \phi, p_{r}, p_{\theta}, p_{\phi}\right)=p_{r} \sqrt{r(1+\cos \theta)}-p_{\theta} \sqrt{\frac{1-\cos \theta}{r}} \\
& \mathcal{B}\left(r, \theta, \phi, p_{r}, p_{\theta}, p_{\phi}\right)=p_{r} \sqrt{r(1-\cos \theta)}+p_{\theta} \sqrt{\frac{1+\cos \theta}{r}} \\
& \mathcal{C}\left(r, \theta, \phi, p_{r}, p_{\theta}, p_{\phi}\right)=p_{\phi}+r(1+\cos \theta)\left(\frac{2 m k}{\hbar(N+2)}+\frac{\mu}{r}\right) \\
& \mathcal{D}\left(r, \theta, \phi, p_{r}, p_{\theta}, p_{\phi}\right)=p_{\phi}-r(1+\cos \theta)\left(\frac{2 m k}{\hbar(N+2)}-\frac{\mu}{r}\right) \\
& \mathcal{E}\left(r, \theta, \phi, p_{r}, p_{\theta}, p_{\phi}\right)=p_{\phi}+r(1-\cos \theta)\left(\frac{2 m k}{\hbar(N+2)}-\frac{\mu}{r}\right) \\
& \mathcal{F}\left(r, \theta, \phi, p_{r}, p_{\theta}, p_{\phi}\right)=p_{\phi}-r(1-\cos \theta)\left(\frac{2 m k}{\hbar(N+2)}+\frac{\mu}{r}\right) \\
& \tilde{\mathcal{A}}\left(\tilde{r}, \tilde{\theta}, \tilde{\phi}, p_{\tilde{r}}, p_{\tilde{\theta}}, p_{\tilde{\phi}}\right)=p_{\tilde{r}} \sqrt{\tilde{r}(1-\cos \tilde{\theta})}+p_{\tilde{\theta}} \sqrt{\frac{1+\cos \tilde{\theta}}{\tilde{r}}} \\
& \tilde{\mathcal{B}}\left(\tilde{r}, \tilde{\theta}, \tilde{\phi}, p_{\tilde{r}}, p_{\tilde{\theta}}, p_{\tilde{\phi}}\right)=p_{\tilde{r}} \sqrt{\tilde{r}(1+\cos \tilde{\theta})}-p_{\tilde{\theta}} \sqrt{\frac{1-\cos \tilde{\theta}}{\tilde{r}}}
\end{aligned}
$$




$$
\begin{aligned}
& \tilde{\mathcal{C}}\left(\tilde{r}, \tilde{\theta}, \tilde{\phi}, p_{\tilde{r}}, p_{\tilde{\theta}}, p_{\tilde{\phi}}\right)=p_{\tilde{\phi}}-\tilde{r}(1-\cos \tilde{\theta})\left(\frac{2 m k}{\hbar(N+2)}+\frac{\mu}{\tilde{r}}\right) \\
& \tilde{\mathcal{D}}\left(\tilde{r}, \tilde{\theta}, \tilde{\phi}, p_{\tilde{r}}, p_{\tilde{\theta}}, p_{\tilde{\phi}}\right)=p_{\tilde{\phi}}+\tilde{r}(1-\cos \tilde{\theta})\left(\frac{2 m k}{\hbar(N+2)}-\frac{\mu}{\tilde{r}}\right) \\
& \tilde{\mathcal{E}}\left(\tilde{r}, \tilde{\theta}, \tilde{\phi}, p_{\tilde{r}}, p_{\tilde{\theta}}, p_{\tilde{\phi}}\right)=p_{\tilde{\phi}}-\tilde{r}(1+\cos \tilde{\theta})\left(\frac{2 m k}{\hbar(N+2)}-\frac{\mu}{\tilde{r}}\right) \\
& \tilde{\mathcal{F}}\left(\tilde{r}, \tilde{\theta}, \tilde{\phi}, p_{\tilde{r}}, p_{\tilde{\theta}}, p_{\tilde{\phi}}\right)=p_{\tilde{\phi}}+\tilde{r}(1+\cos \tilde{\theta})\left(\frac{2 m k}{\hbar(N+2)}+\frac{\mu}{\tilde{r}}\right) .
\end{aligned}
$$

Furthermore we have the equalities

$$
\tilde{\mathcal{A}}=\mathcal{A}, \quad \tilde{\mathcal{B}}=\mathcal{B}, \quad \tilde{\mathcal{C}}=-\mathcal{C}, \quad \tilde{\mathcal{D}}=-\mathcal{D}, \quad \tilde{\mathcal{E}}=-\mathcal{E}, \quad \tilde{\mathcal{F}}=-\mathcal{F}
$$

leading to the following equivalence

$$
f_{N}\left(r, \theta, \phi, p_{r}, p_{\theta}, p_{\phi}\right)=f_{N}\left(\tilde{r}, \tilde{\theta}, \tilde{\phi}, p_{\tilde{r}}, p_{\tilde{\theta}}, p_{\tilde{\phi}}\right) .
$$

\section{Acknowledgements}

The author would like to thanks to organizers of the XIV International Conference Geometry, Integrability and Quantization for giving her the opportunity to talk abroad about her study. Especially she is grateful to Professor I. Mladenov for having the kindness to complete the arrangements for not to get lost in her first foreign trip as sure as to Professor A. Yoshioka for undertaking the task of guiding her Ph.D. research.

\section{References}

[1] Iwai T., On a "Conformal" Kepler Problem and Its Reduction, J. Math. Phys. 22 (1981) 1633-1639.

[2] Iwai T. and Uwano Y., The Four-dimensional Conformal Kepler Problem Reduces to the Three-dimensional Kepler Problem with a Centrifugal Potential and Dirac's Monopole Field. Classical Theory, J. Math. Phys. 27 (1986) $1523-1529$.

[3] Iwai T. and Uwano Y., The Quantised MIC-Kepler Problem and Its Symmetry Group for Negative Energies, J. Phys. A: Math. \& Gen. 21 (1988) 4083-4104.

[4] Kanazawa T., A Specific Illustration of Section Derived From *-unitary Evolution Function, In: New Developments in Geometric Mechanics, T. Iwai (Ed), RIMS, Kyoto 2012, pp 172-191. 
[5] Kanazawa T. and Yoshioka A., Star Product and Its Application to the MICKepler Problem, J. Geom. Symmetry Phys. 25 (2012) 57-75.

[6] Mladenov I. and Tsanov V., Geometric Quantisation of the MIC-Kepler Problem, J. Phys. A: Math. \& Gen. 20 (1987) 5865-5871.

Tomoyo Kanazawa

Department of Mathematics

Graduate School of Science

Tokyo University of Science

Kagurazaka1-3, Shinjuku-ku

Tokyo 162-8601, JAPAN

E-mail address: tomo-n-jewel@qit.ne.jp 